\title{
Periodic Steady-State Analysis of Coupled ODE-AE-CGE Systems for MOS RF Autonomous Circuit Simulation
}

\author{
ASIC and System State Key Lab \\ Fudan University \\ Shanghai, P.R.China, 200433 \\ Tel: +86-021-65643659 \\ Fax: +86-021-65644128 \\ e-mail: jmlai@fudan.edu.cn
}

Xinyu Wu, Zaiman Chen, Jinmei Lai, Qianling Zhang, Omar Wing, Junyan Ren

\begin{abstract}
This paper studies the steady-state analysis of a system of ODE-AE-CGE arising from simulation of MOS RF autonomous circuits in which the transistor plays as current generator and its current equations could adequately represent the transistor large-signal dynamic behavior. Gauss-Seidel relaxation is applied to decouple the system so that shooting method, which is used to find the periodic steady-state response, can be performed with low-order sensitivity matrix. This simplifies the solution process and results in a fast algorithm. We illustrate the new algorithm with the simulation of a typically voltage-controlled oscillator (VCO), the simulation results show good agreement with those obtained by MEDICI, a device simulator.
\end{abstract}

\section{INTRODUCTION}

In MOS RF circuit design, it is crucial to be able to accurately predict the performance of circuits in order to reduce design cycles and have first success. However, the simulation of RF circuits with available MOS compact models often gives inaccurate or even erroneous results [1]. To adequately represent a transistor for large-signal dynamic operations in the $\mathrm{GHz}$ frequency range, a description based on first principles of device physics seems essential. Such a description requires that the transistor be modeled by a system of partial differential equations (PDE) [2-5]. Iterative methods have been developed to compute the transient response of RF circuits with a PDE model of the transistors [2,3]. The algorithm is effective, but the computation time is long and convergence is often slow or not at all. Moreover, in RF circuit design, it is more useful to examine the periodic steady state response than the transient response. Current computation methods exist to find the periodic steady state response of circuits usually described by ordinary differential equations (ODE) [6-10]. Although there are few methods used to simulate the circuits founded on the device simulator in which the devices are described by PDE, they are very inefficient and not practicality for circuit simulation $[11,12]$.

In this paper, we focus on the autonomous circuit and its pe- riodic steady state analysis. The circuit equations consist of ODE for the passive part of the circuit, algebraic equations (AE) if there are circuit nodes to which not any energy storage element is connected, and PDE for the MOS transistors [3]. The problem seems formidable. However, in common oscillator, the voltage waveforms and the period are largely determined by the parameters of the passive part [1]. The transistors serve only as current generators that supply currents to overcome the loss in the resonant circuit. So, we regard the transistor as a multiterminal voltage-dependent current source. To accelerate the computation speed, such current source can be described by a set of current ordinary differential equations [5], it is an adequate representation of the PDE model. We call those current equations as current generate equations (CGE). In fact, the CGE is the functions of the state variables, which include the node voltages of the transistors.

To find the periodic steady state response of such an ODEAE-CGE system, we first apply Gauss-Seidel relaxation [13] to decouple the three sets of equations, it gives a universal way to solve the coupled ODE-AE-CGE system. Shooting method is applied to the circuit ODE only, with the transistor currents computed from the lumped model at each iteration. The pseudo-inverse method [7] is used to update the initial value of the state variables and the period, which gives an efficient way to find the unknown period in oscillator and enhances the stability of shooting iteration either. Performing the transient analysis for a few cycles at the beginning make it easy to select good initial guesses.

We illustrate the new algorithm in the solution of a differential MOS VCO, which has one algebraic node. The results compare well with those obtained by transient analysis based on a PDE model of the transistors [3]. Good consistency is also presented comparing with device simulators such as MEDICI.

The contents of this paper are organized as follows: In Section 2, the circuit equations are derived. The solution algorithm is described in Section 3. In Section 4, simulation results and the convergence comparison are presented. A summary at the end concludes the paper.

\footnotetext{
* This research is supported by the Shanghai Applied Materials R\&D Fund (0002).
} 


\section{EQUATIONS OF COUPLED ODE-AE-CGE SYSTEM}

In our formulation, the transistors are represented by a mathematical description or a procedure by which the transistor currents can be computed once the terminal voltages are given. Transistor models could be described by a system of partial differential equations (PDE) [2-4] or other mathematical description.

It seems natural to describe the passive part of a circuit separately from the nonlinear elements. We write equations for the passive part in the form:

$$
\boldsymbol{M} \frac{d \boldsymbol{X}(t)}{d t}=\boldsymbol{f}(\boldsymbol{X}(t))
$$

where $\boldsymbol{X}(t)$ is a vector of node voltages and inductor currents, $\boldsymbol{M}$ is constant matrix.

If there are nodes in the circuit which are not connected to any dynamic elements, these nodes are named as algebraic nodes. Node equations of algebraic nodes are algebraic, which can be represented by:

$$
\boldsymbol{f}_{A E}\left(\boldsymbol{I}_{\boldsymbol{D}}^{\prime}(t), \boldsymbol{i}_{\boldsymbol{L}}^{\prime}(t)\right)=0
$$

where $\boldsymbol{I}_{\boldsymbol{D}}^{\prime}(t)$ is a subset of the transistor currents set and $\boldsymbol{i}_{\boldsymbol{L}}^{\prime}(t)$ is a subset of the inductor currents set.

Under large-signal operation, it is useful to think of the transistors as current generators. They provide the currents to the passive part of the circuit. The PDE model describes the transistor continuously, but the simulation time is long and convergence is often a problem [3]. Lumped model is another mathematical description of transistor which is less complicated than the PDE model and is sufficiently accurate for RF circuit simulation [5]. No matter what mathematical model is selected, transistor will be described by a set of transistor equations, which can be wrote as the following CGEs:

$$
\boldsymbol{f}_{C G E}\left(\boldsymbol{v}_{\boldsymbol{s}}(t), \boldsymbol{v}_{\boldsymbol{d}}(t), \boldsymbol{v}_{g}(t), \boldsymbol{i}_{\boldsymbol{s}}(t), \boldsymbol{i}_{\boldsymbol{d}}(t), \boldsymbol{i}_{g}(t)\right)=0
$$

Given the transistor drain voltage $v_{d}(t)$, source voltage $v_{s}(t)$ and gate voltage $\boldsymbol{v}_{g}(t)$, its drain current $\boldsymbol{i}_{\boldsymbol{d}}(t)$, source current $\boldsymbol{i}_{\boldsymbol{s}}(t)$ and gate current $\boldsymbol{i}_{g}(t)$ can be found from the CGEs. We assume all the bulk terminals are grounded.

We have the equations of coupled ODE-AE-CGE system form Eq.(1) to Eq.(3) now.

\section{SIMULATION ALGORITHM}

\section{A. Shooting Newton Method}

Shooting method is a popular method to find the initial values which would lead to a periodic steady-state solution [6,7] and can be determine the unknown system period by use of pseudo-inverse expediently $[7,10]$.

Consider the system of equations

$$
\frac{d \boldsymbol{X}}{d t}=\boldsymbol{f}(\boldsymbol{X})
$$

$$
\boldsymbol{X}(0)-\boldsymbol{X}(\boldsymbol{X}(0), T)=0
$$

where $\boldsymbol{X}(t)$ is an $n$-dimensional vector of circuit state variables. Eq.(5) is the periodic boundary condition. Generally, $\boldsymbol{X}(T)$ is an implicit function of the initial value $\boldsymbol{X}(0)$, and it will be denoted as $\boldsymbol{X}(\boldsymbol{X}(0), T)$, which is computed from Eq.(4) using a suitable initial value problem solver.

For an autonomous system, the period $T$ is an unknown. Starting from an initial guess of $\boldsymbol{X}(0)$ and $T$, we generate the next iterate from Eq.(6), as described in [7]. (Superscript " $k$ " indicates the iteration order).

$$
\left[\begin{array}{c}
\boldsymbol{X}^{k+1}(0) \\
T^{k+1}
\end{array}\right]=\left[\begin{array}{c}
\boldsymbol{X}^{k}(0) \\
T^{k}
\end{array}\right]-\left[\boldsymbol{J}_{\boldsymbol{E x t}}\right]^{+}\left[\boldsymbol{X}^{k}(0)-\boldsymbol{X}^{k}(T)\right]
$$

where $\left[\boldsymbol{J}_{\boldsymbol{E} x t}\right]^{+}$is the Moore-Penrose pseudo-inverse of $\boldsymbol{J}_{\boldsymbol{E} \boldsymbol{x}}$, and $\boldsymbol{J}_{E x t}$ is a $n$ by $n+1$ extended matrix with $n$ rank.

$$
\boldsymbol{J}_{E x t}=\left[\boldsymbol{I}-\frac{\partial \boldsymbol{X}(\boldsymbol{X}(0), T)}{\partial \boldsymbol{X}(0)},-\boldsymbol{f}(\boldsymbol{X}, T)\right]
$$

where $\frac{\partial \boldsymbol{X}(\boldsymbol{X}(0), T)}{\partial \boldsymbol{X}(0)}$ is the sensitivity matrix, $\boldsymbol{I}$ is the identity

matrix, and $\boldsymbol{X}^{k+1}(0)$ is the initial value for iteration $k+1$. The sensitivity matrix can be calculated by solving the initial value problem of the variational differential equation of Eq.(4) $[6,10]$.

Since $\boldsymbol{J}_{E x t}$ has more columns than rows and is not of full rank, the pseudo-inverse does not have a unique solution. Moore-Penrose pseudo-inverse gives the smallest result than any other solution to approach the norm $\left\|\left[\boldsymbol{J}_{\boldsymbol{E x t}}\right]^{+} \boldsymbol{J}_{\boldsymbol{E x t}}-\boldsymbol{I}\right\|$. Experiments show that the pseudo-inverse method is more stable than the substitute method in [6].

\section{B. Iteration Algorithm}

It can be seemed as a current generation procedure that solve the current generator equations. We illuminate this procedure by use of the lumped model of MOSFET in appendix:

1) Given the drain, source and gate voltages, determine the boundary conditions of the surface potentials at the source and drain, $u(0, t)$ and $u(L, t)$, from boundary equation [Eq (A.9) and Eq.(A.10)] respectively, with $L$ being the length of the channel.

2) Specify the initial condition of the surface potential $u(x, 0)$ along the channel.

3) Solve transistor equations [Eq.(A.4) to Eq.(A.6)] for $t \in\left[0, t_{\max }\right]$, where $t_{\max }$ is equal to the period $T$ in the steady state analysis.

4) Compute the source and drain current $i_{s}(t), i_{d}(t)$ from current equations [Eq.(A.7) and Eq.(A.8)] respectively

Using shooting method to find the periodic steady state response of coupled ODE-AE-CGE system directly, the sensitivity matrix will be hard to calculate. By consider transistor as current generator, we can take the transistors out of the calculation of sensitivity matrix, update the initial values of circuit 
variables without considering transistors. Since the terminal voltages are included in the set of circuit variables, CGEs will renew the terminal currents further more. The updating in shooting method then can be decomposed into two steps: the renewal of initial values of circuit variables and the rebirth of terminal currents followed.

Now, we rewrite the three sets of circuit equations, the passive $\mathrm{ODE}, \mathrm{CGE}$ and $\mathrm{AE}$, as functions of transistor currents, algebraic node voltages, and state variables. Included in iteration of shooting method, the terminal voltages of transistors will be updated by solving ODE while the terminal currents will be updated by solving CGE. Gauss-Seidel decoupling [13] illuminates this procedure properly:

$$
\begin{array}{r}
\boldsymbol{f}_{O D E}\left(\boldsymbol{X}^{k+1}(t), T^{k+1}, \boldsymbol{i}_{s}^{k}(t), \boldsymbol{i}_{d}^{k}(t), \boldsymbol{i}_{g}^{k}(t), \boldsymbol{X}^{k}(0), T^{k}\right)=0 \\
\boldsymbol{f}_{A E}\left(\boldsymbol{X}^{k+1}(t), T^{k+1}, \boldsymbol{i}_{s}^{k+1}(t), \boldsymbol{i}_{d}^{k}(t), \boldsymbol{i}_{g}^{k}(t), \boldsymbol{v}_{A E}^{k+1}(t), \boldsymbol{v}_{A E}^{k}(t)\right)=0 \\
\boldsymbol{f}_{C G E}\left(\boldsymbol{X}^{k+1}(t), T^{k+1}, \boldsymbol{i}_{s}^{k+1}(t), \boldsymbol{i}_{d}^{k+1}(t), \boldsymbol{i}_{g}^{k+1}(t), \boldsymbol{v}_{A E}^{k+1}(t)\right)=0
\end{array}
$$

where $\boldsymbol{X}^{k}(t), \boldsymbol{i}_{d}^{k}(t), \boldsymbol{i}_{s}^{k}(t), \boldsymbol{i}_{g}^{k}(t)$ and $\boldsymbol{v}_{\boldsymbol{A E}}^{k}(t)$ are vectors of waveforms of circuit state variables, drain current, source current, gate current and algebraic node voltage respectively, $T^{k}$ is the system period. Eq.(8) can be solved one by one.

This scheme combines the shooting Newton method with Gauss-Seidel relaxation between the ODE, AE and CGE. The detailed algorithm is as follows:

1) Obtain a periodic initial guess of the drain, source and gate current waveforms $\boldsymbol{i}_{d}^{0}(t), \boldsymbol{i}_{s}^{0}(t)$ and $\boldsymbol{i}_{g}^{0}(t)$, respectively, $t \in\left[0, T^{0}\right]$, where $T^{0}$ is the initial guess of the system period.

2) Consider each transistor as a current generator. Solve the ODE of the passive part with initial value $\boldsymbol{X}^{k}(0)$ and get the waveforms $\boldsymbol{X}^{k}(t)$ of states, $t \in\left[0, T^{k}\right]$.

3) Compute the sensitivity matrix and update the initial value of states to $\boldsymbol{X}^{k+1}(0)$ and period to $T^{k+1}$ from Eq.(6).

4) Update the drain, source and gate voltage waveforms to $\boldsymbol{v}_{d}^{k+1}(t), \boldsymbol{v}_{s}^{k+1}(t)$ and $\boldsymbol{v}_{g}^{k+1}(t)$ from the waveforms of states $\boldsymbol{X}^{k}(t)$.

5) Solve the AE, and update $\boldsymbol{v}_{A E}^{k}(t)$ to $\boldsymbol{v}_{A E}^{k+1}(t)$.

6) Follow the current generator procedure to obtain the transistor currents. Update $\boldsymbol{i}_{d}^{k}(t), \boldsymbol{i}_{s}^{k}(t)$ and $\boldsymbol{i}_{g}^{k}(t)$.

7) Return to step 2 until all the Euclidean error norms of the current and voltage waveforms at the last iteration are less than a specified tolerance, and all of the relative errors of boundary values are less than a given tolerance.

To find a good initial guess of the shooting points and system period as we start the solution, transient analysis is used in the beginning.

\section{SiMULATION RESULTS}

We apply the new algorithm to compute the periodic steady state response of a typical voltage controlled oscillator (VCO)

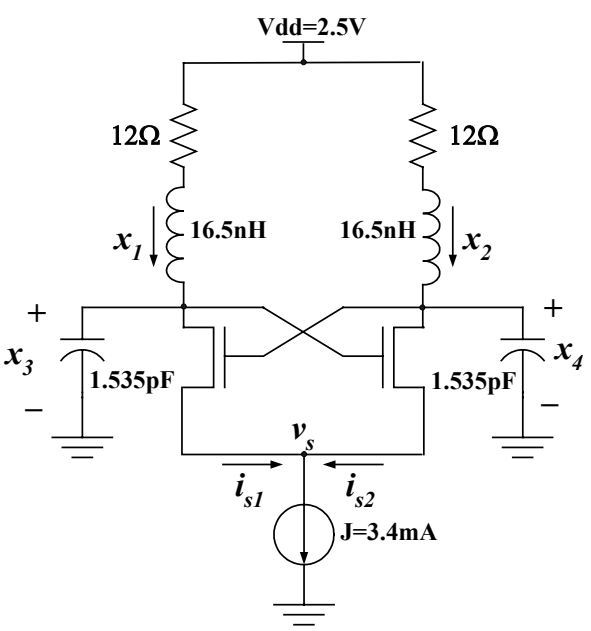

Fig.1. A voltage controlled oscillator example.

circuit [14] shown in Fig.1.

In addition to the ODE and CGE of the transistors, there is an algebraic equation at node $v_{s}$ in this circuit:

$$
f_{A E}\left(v_{s}(t)\right)=i_{s 1}(t)+i_{s 2}(t)-J=0
$$

where $J$ is a constant current source. Since both $i_{s 1}$ and $i_{s 2}$ are functions of $v_{s}(t)$ when the drain voltages have been determined in each iteration, we regard this equation as a nonlinear algebraic equation of the unknown $v_{s}(t)$. We solve this equation by Newton iteration. Assume a starting waveform $v_{s}^{0}(t)$, together with the drain voltages obtained from the solution of the ODE. We find the drain and source currents by the current generator procedure. The next iterate of $v_{s}$ is

$$
v_{s}^{k+1}(t)=v_{s}^{k}(t)-f_{A E}^{\prime}\left(v_{s}^{k}(t)\right)^{-1} f_{A E}\left(v_{s}^{k}(t)\right), \quad t \in[0, T]
$$

where $f_{A E}^{\prime}\left(v_{s}^{k}(t)\right)$ is the Jacobian matrix of $f_{A E}\left(v_{s}^{k}(t)\right)$ with respect to $v_{s}$, which can be readily calculated by use of finite differences.

Transient analysis is used in the beginning for five periods in order to get a good initial guess of the shooting points and system period. We choose zero current in the inductors and 2.5 volt voltage on the capacitors as initial value for the transient analysis. This heuristic scheme makes it easy to build up the oscillation.

We tested the algorithm with different combinations of initial guesses. Some representative initial guesses are: $T^{0} \in[0.90$ $\left.\begin{array}{llll}0.99 & 1.00 & 1.01 & 1.10\end{array}\right] \mathrm{ns}, v_{s}^{0} \in\left[\begin{array}{lllll}0 & 0.5 & 1.0 & 1.5 & 2.0\end{array}\right]$ volt, and $i_{d 1}^{0} \in\left[\begin{array}{lllll}0.001 & 0.5 & 1.0 & 1.5 & 1.7\end{array}\right] \mathrm{mA}$. A tolerance of $1 \times 10^{-3}$ is set and the Euclidean norm of the error versus iteration number is plotted in Fig.2. In most cases, convergence occurs after about 20 iterations, and they exhibit fast convergent rate as shown. The best case occurs when we give $T^{0}=1.10 \mathrm{~ns}, v_{s}^{0}=2.0$ volt and $i_{d 1}^{0}=0.5 \mathrm{~mA}$, while the worst case occurs at $T^{0}=0.90 \mathrm{~ns}$, $v_{s}^{0}=0.5$ volt and $i_{d 1}^{0}=1.5 \mathrm{~mA}$. Both cases return the same waveforms and system period $T^{*}=1.010 \mathrm{~ns}$.

A good initial guess of the period is critical in solving an autonomous system. The theoretic period of this circuit is 


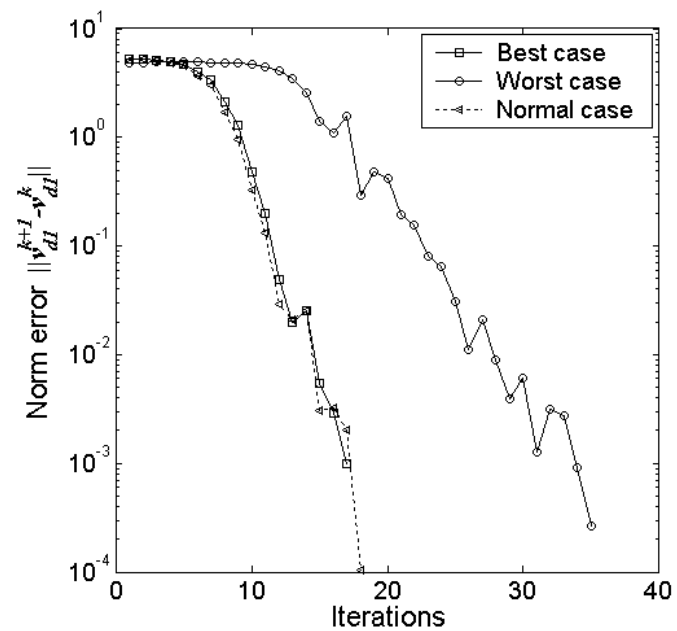

Fig.2. Euclidean norm of the error versus iterations.

1.0067 ns. We vary initial guess value of the period from $0.90 \mathrm{~ns}$ to $1.10 \mathrm{~ns}$. For each guess, we select $v_{s}^{0}(t)$ from 0 to 2.0 volt while $i_{d 1}^{0}(t)$ varies from 0 to $1.7 \mathrm{~mA}$. Twenty-five cases have been tested. Table I gives a summary of convergence rate for different initial choices of period $T^{0}$.

The effect of initial guess on convergence of the algebraic node voltage $v_{s}(t)$ is illustrated in Table II. We find convergence is not sensitive to the source voltage waveform. The steady state analysis will be successfully completed even if we give a constant DC value to $v_{s}^{0}(t)$, which varies from 0 to 2.0 volt.

TABLE I

Initial Guess of System Period*

\begin{tabular}{|c|c|c|c|c|}
\hline \multirow{2}{*}{$\begin{array}{c}\text { Initial guess } \boldsymbol{T}^{\boldsymbol{0}} \\
(\mathrm{sec})\end{array}$} & \multicolumn{2}{|c|}{$\begin{array}{c}\text { Number of } \\
\text { iterations }\end{array}$} & \multicolumn{2}{c|}{ CPU Time (sec) } \\
\cline { 2 - 5 } & Min & $\max$ & $\min$ & $\max$ \\
\hline $0.90 \times 10^{-9}$ & 24 & 36 & 138 & 282 \\
\hline $0.99 \times 10^{-9}$ & 19 & 35 & 104 & 251 \\
\hline $1.00 \times 10^{-9}$ & 19 & 32 & 104 & 274 \\
\hline $1.01 \times 10^{-9}$ & 18 & 31 & 102 & 211 \\
\hline $1.10 \times 10^{-9}$ & 18 & 30 & 95 & 134 \\
\hline
\end{tabular}

$* v_{s}^{0} \in\left[\begin{array}{lllll}0 & 0.5 & 1.0 & 1.5 & 2.0\end{array}\right]$ volt, $i_{d 1}^{0} \in\left[\begin{array}{lllll}0.001 & 0.5 & 1.0 & 1.5 & 1.7\end{array}\right] \mathrm{mA}$.

TABLE II

INITIAL GuesS OF SOURCE Voltage WAVEForm*

\begin{tabular}{|c|c|c|c|}
\hline $\begin{array}{c}\text { Initial guess } \\
\boldsymbol{v}_{\boldsymbol{s}}^{\boldsymbol{v}}(\boldsymbol{t}) \text { (volt) }\end{array}$ & $\begin{array}{c}\text { Number of } \\
\text { Iterations }\end{array}$ & $\begin{array}{c}\text { CPU Time } \\
(\mathrm{sec})\end{array}$ & Convergence \\
\hline 0.0 & 19 & 106 & Yes \\
\hline 0.5 & 19 & 105 & Yes \\
\hline 1.0 & 19 & 104 & Yes \\
\hline 1.5 & 19 & 104 & Yes \\
\hline 2.0 & 19 & 104 & Yes \\
\hline
\end{tabular}

*Initial guesses are $T^{0}=1.00 \times 10^{-9} \mathrm{sec}, i_{d 1}^{0}(t)=1 \times 10^{-6} \mathrm{Amp}$
Convergence is mildly sensitive to the initial guess of $i_{d 1}^{0}(t)$ as revealed in Table III. When the initial current waveforms in the two transistors are given almost equal values, e.g., $i_{d 1}^{0}(t)=1.7 \mathrm{~mA}$ or $i_{d 1}^{0}(t)=1.5 \mathrm{~mA}$, the number of iterations increases significantly.

TABLE III

INITIAL Guess of Drain CURRENT WAVEForm*

\begin{tabular}{|c|c|c|c|}
\hline $\begin{array}{c}\text { Initial guess } \\
\boldsymbol{i}_{\boldsymbol{d} \boldsymbol{0}}(t)(\text { Amp })\end{array}$ & $\begin{array}{c}\text { Number of } \\
\text { Iteration }\end{array}$ & $\begin{array}{c}\text { CPU Time } \\
(\mathrm{sec})\end{array}$ & Convergence \\
\hline $1 \times 10^{-6}$ & 18 & 101 & Yes \\
\hline $0.5 \times 10^{-3}$ & 19 & 105 & Yes \\
\hline $1.0 \times 10^{-3}$ & 20 & 108 & Yes \\
\hline $1.5 \times 10^{-3}$ & 22 & 115 & Yes \\
\hline $1.7 \times 10^{-3}$ & 31 & 211 & Yes \\
\hline
\end{tabular}

*Initial guesses are $T^{0}=1.01 \times 10^{-9} \mathrm{sec}, v_{s}^{0}(t)=1.0 \mathrm{volt}$

Finally the waveforms of the drain and source voltages are given in Fig.3. The drain and source currents are plotted in Fig.4. We use solid lines to indicate the waveforms obtained by the present method based on the lumped model, while we use dash-dot lines to indicate the results obtained from transient analysis based on the PDE model [3]. Presented method show good agreements with transient method [3], while the former is much faster than later, which will cost hours to get steady-state response. Further more, the former exhibit good convergence.

We also tested our results against MEDICI [15], which is a device simulator and is able to perform transient analysis for simple circuit. The results obtained from MEDICI are indicated by dotted lines. They are also compares well with the presented method, but MEDICI is more complicated and the simulation time is always insufferable. The maximum relative error of presented method compared with MEDICI is less than $7 \%$ as show in Fig.4.

\section{CONCLUSION}

We have presented an efficient iterative algorithm for determination of the periodic response and the period of coupled

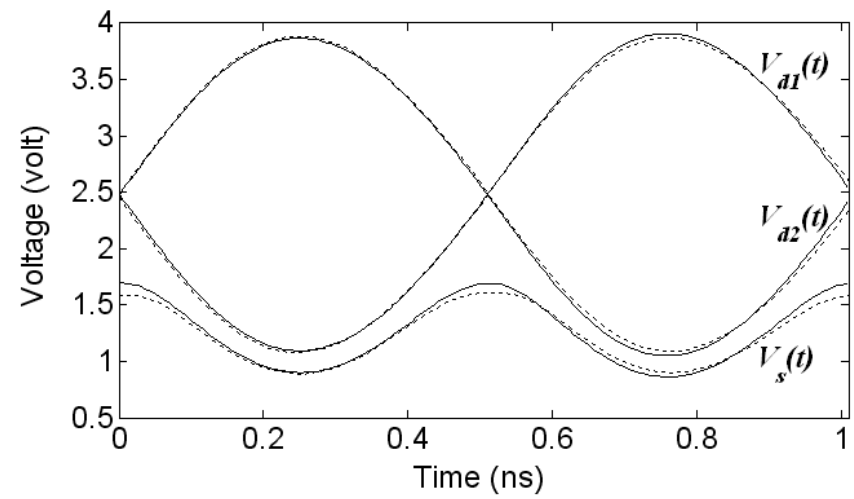

Fig. 3. Drain and source voltage waveforms by presented method (solid line), transient method [3] (dotted line) and MEDICI (dash-dot line). 


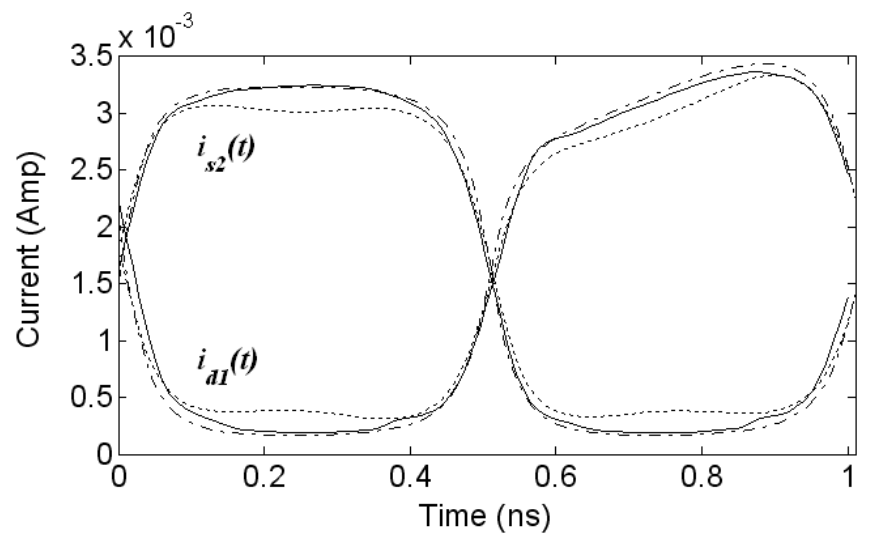

Fig.4. Drain and source current waveforms by presented method (solid line), transient method [3] (dotted line) and MEDICI (dash-dot line).

ODE-AE-CGE system arising from simulation of MOS RF autonomous circuits.

Gauss-Seidel relaxation decouples the system that described by three sets of equations, and makes it possible to solve these equations separately. Shooting method combined with the GS relaxation form a scheme to update initial values easily by calculating low-order sensitivity matrix and renew terminal currents. As a result, iteration by the use of pseudo-inverse is over a low order system and convergence is relatively fast.

\section{ACKNOWLEDGMENTS}

The authors wish to thank Professor Chenshou Sun and Professor Jun Xu for their support and encouragement.

\section{APPENDIX: LUMPED MODEL OF THE MOSEFET}

Since reference [5] may not be widely available, we summarize the current generator equations of lumped model below. Similar versions of most of these equations are found in [3] and [5]. See text for definition of the terms.

Fig. 5 shows a lumped model use to approximate the PDE description of the transistor. The channel is divided into a small number of segments, each described by two nonlinear lumped elements: a nonlinear conductance and a nonlinear charge "store."

In following equations, $u(x, t)$ is the surface potential; $\mu$ is the mobility; $v_{t}$ is the thermal voltage; $V_{F B}$ is the flat band voltage; $V_{D P}$ is a voltage related to the doping concentration; $v_{g b}(t)$ is the gate voltage; $W$ is the channel width; and $C_{o x}$ is the oxide capacitance.

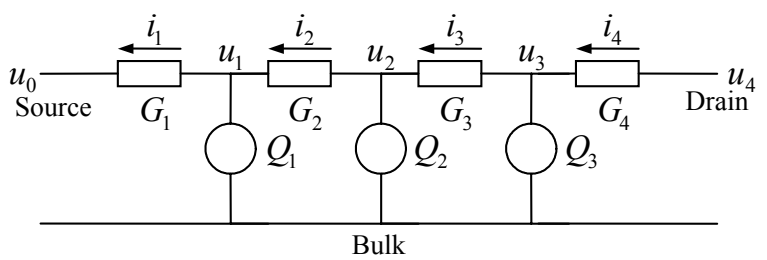

Fig.5. A proposed lumped model.
Surface potentials at the segment points be $u_{1}, u_{2}, u_{3}$ and $u_{4}$, with $u_{0}=u(0, t)$ and $u_{4}=u(L, t)$ being the surface potentials at the source and drain.

The nonlinear conductance is

$$
G_{k}=\frac{1}{2 l_{k}}\left(G\left(u_{k}\right)+G\left(u_{k-1}\right)\right), \quad k=1,2,3,4
$$

The lumped charge is

$$
Q_{k}=u C_{o x} \frac{W}{I_{D C}}\left[q\left(u_{k}\right) \frac{d x}{d u}\left|u_{k}+q\left(u_{k-1}\right) \frac{d x}{d u}\right| u_{k-1}\right]\left(u_{k}-u_{k-1}\right)
$$

where

$$
\begin{aligned}
\frac{d x}{d u}= & u C_{o x} \frac{W}{I_{D C}}\left[v_{g b}-u-V_{F B}-\right. \\
& \left.-V_{D P} \sqrt{e^{-u / v_{t}}-1+u / v_{t}}+\frac{1}{2} V_{D P} \frac{1-e^{-u / v_{t}}}{\sqrt{e^{-u / v_{t}}-1+u / v_{t}}}\right]
\end{aligned}
$$

Circuit equations are

$$
\begin{aligned}
& \frac{d Q_{1}}{d t}=G_{2} u_{2}-\left(G_{2}+G_{1}\right) u_{1}+G_{1} u_{0} \\
& \frac{d Q_{2}}{d t}=G_{3} u_{3}-\left(G_{3}+G_{2}\right) u_{2}+G_{2} u_{1} \\
& \frac{d Q_{3}}{d t}=G_{4} u_{4}-\left(G_{4}+G_{3}\right) u_{3}+G_{3} u_{2}
\end{aligned}
$$

The drain and source current can be found from

$$
\begin{aligned}
& i_{s}(t)=G_{1}\left(u_{1}-u_{0}\right) \\
& i_{d}(t)=G_{4}\left(u_{4}-u_{3}\right)
\end{aligned}
$$

The surface potentials $u_{0}=u(0, t)$ and $u_{4}=u(L, t)$ at the source and drain are given by the following two implicit equations

$$
\begin{gathered}
v_{g}(t)-V_{F B}-u(0, t)-V_{D P}\left(e^{-u(0, t) / v_{t}}-1+u(0, t) / v_{t}+\right. \\
\left.+e^{-2 v_{f} / v_{t}}\left(e^{\left(u(0, t)-v_{s}(t)\right) / v_{t}}-e^{-v_{s}(t) / v_{t}}-u(0, t) / v_{t}\right)\right)^{1 / 2}=0 \\
v_{g}(t)-V_{F B}-u(L, t)-V_{D P}\left(e^{-u(L, t) / v_{t}}-1+u(L, t) / v_{t}+\right. \\
\left.\quad+e^{-2 v_{f} / v_{t}}\left(e^{\left(u(L, t)-v_{d}(t)\right) / v_{t}}-e^{-v_{d}(t) / v_{t}}-u(L, t) / v_{t}\right)\right)^{1 / 2}=0
\end{gathered}
$$

At last, gate current can be found form following equation

$$
i_{g}(t)=W L C_{o x} \frac{d}{d t}\left[v_{g}(t)-V_{m s}-\frac{1}{L} \int_{0}^{L} u(x, t) d x\right]
$$

\section{REFERENCES}

[1] Willy Sansen, Johan Huijsing, Analog circuit design (X)DSL and other communication systems; RF MOST models; Integrated filters and oscillators, Kluwer academic publishers, 1999.

[2] Y.Tsividis, Operation and Modeling of the MOS Transistor. McGraw-Hill, $2^{\text {nd }}$ edition, 1999. 
[3] Omar Wing, Tan Jun, Lai Jinmei, Ren Junyan and Zhang Qianling, "Iterative Solution of ODE-PDE-AE Systems for RF Circuit Simulation”, IEEE ISCAS 2002, May 2002, Scottsdale, Arizona.

[4] Wu Xinyu, Lai Jinmei, Omar Wing, Zhang Qianling, Ren Junyan and Sun Chenshou, "Periodic Steady State Solution of PDE-ODE System for RF Circuit Simulation", IEEE ICCCAS 2002, June 2002, Chengdu, P.R.China.

[5] Chen Zaiman, Lai Jinmei, Omar Wing and Ren Junyan, "A Lumped, Large-Signal Dynamic Model of the MOSFET for RF Circuit Simulation", IEEE ICCSC 2002, June 2002, St.Petersburg, Russia.

[6] T.J.Aprille and T.N.Trick, "A Computer Algorithm to Determine the Steady-State Response of Nonlinear Oscillator", IEEE Trans. Circuit Theory, vol.CT-19, no.4, pp.354-360, Jul, 1972.

[7] J.R.Parkhurst and L.L.Ogborn, "Determining the SteadyState Output of Nonlinear Oscillatory Circuits using Multiple Shooting", IEEE Trans. CAD, vol.14, no.7, pp.882-889, Jul, 1995.

[8] Ken.Kundert, "Simulation of Nonlinear Circuit in the Frequency Domain”, IEEE Trans. CAD, vol.CAD-5, no.4, pp.521-
535, Oct, 1986.

[9] D.R.Frey. "A Class of Relaxation Algorithms for Finding the Periodic Steady-State Solution in Nonlinear System", IEEE Trans. CASI, vol.4.5, pp.659-663, Jun. 1998.

[10] T.S.Parker and L.O.Chua, Practical Numerical Algorithms for Chaotic Systems, Springer-Verlag, 1989.

[11]B.Troyanovsky, Z.P.Yu, L.So and W.Dutton, "RelaxationBased Harmonic Balance Technique for Semiconductor Device Simulation", IEEE ICCAD 95.

[12] Y.T.Hu, and K.Mayaram, "Periodic Steady-State Analysis for Coupled Device and Circuit Simulation", IEEE SISPAD 2000.

[13]A.R.Newton and A.L.Sanggiovanni-Vincentelli, "Relaxation Based Electrical Simulation", IEEE Trans. CAD, vol.CAD3, no.4, pp:308-330, Oct, 1984.

[14] Thomas H.Lee, The design of CMOS radio-frequency integrated circuits, Chapter 16, Cambridge University Press, 1998.

[15]Avant! MEDICI Two-Dimensional Device Simulation Program, Version 1999.2 User's Manual. July 1999. Avant! Corporation, TCAD Business unit, Fremont, California. 